\title{
Comparison of Tandem and Conventional Mass Spectrometry Using Electron Capture Negative Ionization in the Detection of Chemically Oxidized Dexamethasone in Human Plasma
}

\author{
Kathleen A. Kayganich, Timothy G. Heath, and J. Throck Watson \\ Departments of Chemistry and Biochemistry, Michigan State University, East Lansing, Michigan, USA
}

\begin{abstract}
Dexamethasone, a synthetic steroid, can be oxidized chemically to a ketonic steroid structure that is readily detected by electron capture negative ionization mass spectrometry (ECNI/MS). Previous work from this laboratory has demonstrated that the chemical oxidation procedure provides advantages of low detection limits and high selectivity for detection of oxidized dexamethasone against chemical background that would otherwise interfere with detection of this steroidal drug in biological samples using more conventional methodology. This report describes the extent to which tandem mass spectrometry (MS/MS) can further enhance the selectivity of the oxidation/ECNI methodology for the detection of dexamethasone during the analysis of human plasma and presents evidence that sample introduction by direct inlet probe (DIP) can be used successfully under ECNI conditions. For purposes of comparing the methodologies, the same human plasma samples are analyzed by ECNI, first with detection by conventional mass spectrometry using selected ion monitoring (SIM) and then by MS'MS using selected reaction monitoring (SRM) with sample introduction by the gas chromatographic (GC) inlet and by the DIP. The results indicate that use of the DIP is a viable means of sample introduction for ECNI when sample processing involves the specialized oxidation procedure described herein because the sample matrix does not compete significantly for the thermal electrons in the ion source. Whereas SIM and SRM provide comparable results when sample introduction is achieved by the GC inlet, the MS/MS approach offers the possibility for sample introduction using the DIP, which significantly simplifies and shortens the analysis. (J Am Soc Mass Spectrom 1990, 1, 341-348)
\end{abstract}

$\mathrm{O}$ ur goal is to develop sensitive and selective, yet simple, methodology for the determination of dexamethasone and other synthetic steroidal drugs in physiological fluids. We have demonstrated considerable success in this area by chemically oxidizing dexamethasone to a steroid structure that has an approximate 100-fold increase in electrophilic character over that of the oxidation products of most endogenous steroids [1]. Recent preliminary results from a comparative study of the electron caplure negative ionization (ECNI) response to unoxidized dexamethasone, as the tetra-TMS derivative, by gas-liquid chromatography (GLC) inlet and underivatized dexamethasone by direct inlet probe (DIP), versus that of oxidized dexamethasone show an improvement of at least 25-fold by the latter [2]. The determination of low concentrations of dexamethasone in complex biological matrices, such as plasma, requires sensitive and selective methodology. Although ade-

Address reprint requests to J. Throck Watson, Department of Chemistry, Michigan State University, East Lansing, MI 48824. quately sensitive radioimmunoassays (RIA) for dexamethasone in plasma are available [3], cross-reactivity of the antibody can lead to dubious results. Gas chromatography/mass spectrometry (GC/MS) methods are more specific than RIA but not always as sensitive. The usual approach for GC/MS determination of steroids in plasma involves extraction and purification of the steroids fullowed by derivatization to improve the chromatographic properties and thermal stability of the molecules. The most successful derivatization scheme of those reported for dexamethasone involves the formation of the 11,17,21-tris-trimethylsilyl ether20-enol-trimethylsilyl ether (tetra-TMS). By using selected ion monitoring (SIM) GC/EIMS, Kasuya et al. [4] were able to detect an on-column injection of 100 pg of dexamethasone-tetra-TMS [signal-to-noise (S/N) ratio of 2.51 from $1 \mathrm{~mL}$ of plasma spiked to a concentration of $300 \mathrm{pg} / \mathrm{mL}$. However, extensive isolation procedures, consisting of extraction by $\mathrm{C}-18$ bondedphase reverse-phase cartridge followed by normalphase high-performance liquid chromatography purification, were necessary to eliminate the plasma ma- 
trix interferences. In our hands, the detection limits claimed above could not be reproduced [5]. The methods described here, which use the high electron capture activity of the oxidized analyte for analysis by ECNI/MS, are more sensitive for the determination of dexamethasone in plasma than the Kasuya method. By using SIM and ECNI/MS, we were able to detect an injection of $30 \mathrm{pg}$ of oxidized dexamethasone $(S / N=11)$ from $1 \mathrm{~mL}$ of plasma spiked with $209 \mathrm{pg}$ of dexamethasone and $35 \mathrm{ng}$ of internal standard. In addition, extensive isolation procedures are not required for our procedure [5], owing to the selectivity of the oxidation reaction and the ionization by electron capture.

In an effort to further increase the selectivity of the oxidation-based assay with ECNI, we examined the possibility of using a triple quadrupole mass spectrometer (TQMS) for selected reaction monitoring (SRM). There have been reports of improvements by the tandem mass spectrometry (MS/MS) approach to the detection of targeted compounds using ECNI with sample introduction by GC [6-9]. Recently, a quantitative assay for leukotriene- $B_{4}$ (as the butyldimethylsilyl ether derivative) in synovial fluid was reported [10]. The selectivity of ECNI in combination with MS'MS (i.e., ECNI/SRM), allowed quantitation of lower levels of $\mathrm{LTB}_{4}$ in the matrix than would have been possible using ECNI/SIM with a conventional single-stage mass spectrometer.

This report compares results obtained by conventional mass spectrometry using a gas chromatograph as the inlet with results obtained by a TQMS using either a GC inlet or DIP for the analysis by ECNI/MS/MS of chemically oxidized extracts of human plasma for the determination of dexamethasone. Whereas the results described here indicate that SIM and SRM give comparable results when sample introduction is achieved by the GC inlet, the MS/MS approach does offer the possibility for sample introduction by means of the DIP system, which will significantly simplify and shorten the analysis by mass spectrometry.

\section{Experimental}

\section{Instrumentation}

All data were collected on a Finnigan (San Jose, CA) TSQ-70 (triple-stage quadrupole) mass spectrometer equipped with a Varian (Walnut Creek, CA) 3400 gas chromatograph. Source conditions were established to maximize the abundance of the parent ion, $\mathbf{M}^{-}$, for analyses by MSIMS. The molecular anion of the oxidized dexamethasone was at the greatest absolute abundance when ammonia was used as the ECNI modifying gas at a pressure of about 1 torr. The electron energy was $100 \mathrm{eV}$, and the primary electron beam current was $300 \mu \mathrm{A}$. The ion source temperature was maintained at $100{ }^{\circ} \mathrm{C}$. For analyses by SIM, the ion source was maintained at $120^{\circ} \mathrm{C}$ in order to obtain a greater abundance of the fragment ion, $m / z 310$, which was used for quantitation.
Gas Chromatography. Samples were introduced into the ion source via a DB-5 capillary column $(30 \mathrm{~m} \times 0.25$ $\mathrm{mm}, \mathrm{J} \& W$ Scientific, Folsum, CA) directly interfaced to the mass spectrometer. Helium was used as the carrier gas at a flow rate of $1 \mathrm{~mL} / \mathrm{min}$. The column was operated with a splitless injector and, following an injection, the $\mathrm{GC}$ oven temperature was ramped from 60 or $120^{\circ} \mathrm{C}$ to $260^{\circ} \mathrm{C}$ at $40^{\circ} \mathrm{C} / \mathrm{min}$ and from $260^{\circ} \mathrm{C}$ to $280^{\circ} \mathrm{C}$ at $4^{\circ} \mathrm{C} / \mathrm{min}$.

Direct Probe. Samples were introduced into the ion source via the DIP system on the TSQ-70 instrument. After insertion of the probe, the temperature was ramped from 35 to $200^{\circ} \mathrm{C}$ at a rate of $70^{\circ} \mathrm{C} / \mathrm{min}$ and held at $200{ }^{\circ} \mathrm{C}$ for $2 \mathrm{~min}$.

\section{Collision Energy and Pressure Studies}

The conditions for the MS/MS experiments were optimized by varying both the collision gas pressure and collision energy. Conditions were sought that would provide greatest detection of the daughter ion current at $m / z 310$. For these studies, $3.56 \mathrm{ng}$ of oxidized dexamethasone was introduced by the DIP. The probe was held at $40^{\circ} \mathrm{C}$ for $1 \mathrm{~min}$ and then ramped to 200 ${ }^{\circ} \mathrm{C}$ at $30^{\circ} \mathrm{C} / \mathrm{min}$ and held at $200^{\circ} \mathrm{C}$ for $2 \mathrm{~min}$. This temperature program yielded a level plateau region in the desorption profile over which the total ion current (TIC) was relatively constant. Over the course of this constant TIC region, repetitive daughter ion spectra of $m / z 330$ were obtained at different energies. The parent ion current at $m / z 330$ was selected by the first quadrupole $\left(Q_{1}\right)$, and the third quadrupole $\left(Q_{3}\right)$ was scanned from $m / z 275$ to $m / z 345$ at $0.1 \mathrm{~s} / \mathrm{scan}$. (The full daughter spectrum indicated no fragments below $m / z$ 275.) Consecutive daughter ion scans were acquired at collision energies of $1,3,5,12$, and $25 \mathrm{eV}$; this cycle was repeated many times over the course of the desorption profile. Five scans at each collision energy, collected during the period of relatively constant parent ion current in the desorption profile, were averaged to provide a representative daughter ion mass spectrum. Replicate experiments were performed for each collision gas pressure. This protocol permitted a reliable assessment of the fragmentation and collection efficiency as a function of collision energy at a given pressure of collision gas in $Q_{2}$. An argon pressure of approximately 1.3 mtorr and a collision energy of 4 $\mathrm{eV}$ were chosen for the analysis of plasma extracts by SRM. This unusually low value of collision energy was chosen because it yielded maximal intensity at $m / z 310$ as discussed in the Results and Discussion section.

\section{SIM and SRM Studies}

For the determinations of dexamethasone in plasma by SIM, $Q_{1}$ was operated in the normal mass spectrometric scan mode while $Q_{2}$ and $Q_{3}$ were maintained in the rf-only mode. Ion currents at $m / z$ 339.2, 319.2, 330.2 , and 310.2 were monitored with $0.8-\mathrm{u}$ windows. 
The dwell time was $50 \mathrm{~ms}$ at each mass window. The electron multiplier was set at $1200 \mathrm{~V}$.

For the analyses by SRM, the ion currents corresponding to the reactions $m / z 330 \rightarrow m / z 310$ (for oxidized dexamethasone) and $m / z 339 \rightarrow m / z 319$ (for oxidized ${ }^{13} \mathrm{C}_{6},{ }^{2} \mathrm{H}_{3}$-dexamethasone) were monitored with a 1.0-u window. Dwell times were $50 \mathrm{~ms}$. The electron multiplier was set at $1400 \mathrm{~V}$.

\section{Materials}

Chemicals used for the oxidation procedure include pyridinium chlorochromate (Aldrich Chemical Co., Milwaukee, WI) and anhydrous sodium acetate (J. T. Baker Chemical Co., Phillipsburg, NJ). All solvents used were either analytical or HPLC grade, purchased from Burdick and Jackson Division of Baxter Healthcare Corporation (Muskegon, MI), J. T. Baker Chemical Company, (Phillipsburg, NJ), EM Science (Gibbstown, NJ), and Mallinckrodt, Inc. (St. Louis, MO). Celite analytical filter aid was purchased from Fisher Scientific Co. (Pittsburg, PA). Analytical reagent urea was purchased from Mallinckrodt (St. Louis MO). C-18 PrepSep solid-phase extraction columns (200 mg) were purchased from Fisher Scientific. Silica solid-phase extraction columns ( $500 \mathrm{mg}$ ) were purchased from Burdick and Jackson Division of Baxter Healthcare Corporation (Muskegon, MI). Dexamethasone was purchased from Steraloids, Inc. $1,2,3,4,5,19-{ }^{13} C_{6}, 19,19,19$. ${ }^{2} \mathrm{H}_{3}$-dexamethasone was a gift from Clinton Kilts of the Department of Pharmacology at Duke University. All materials were used without further purification. Silanized glassware was used throughout the procedures.

\section{Methods}

Sample preparation based on the oxidation method consists of three steps: plasma extraction, chemical oxidation, and removal of excess oxidation reagent.

\section{Extraction of Plasma Samples}

Samples consisted of $1.5 \mathrm{~mL}$ of plasma taken from patients at various times following administration of $1 \mathrm{mg}$ of dexamethasone. Before extraction, $35 \mathrm{ng}$ of ${ }^{13} \mathrm{C}_{6},{ }^{7} \mathrm{H}_{3}$-dexamethasone in $50 \mu \mathrm{L}$ of methanol was added to each sample. Solid-phase extraction columns containing $200 \mathrm{mg}$ of $\mathrm{C}-18$ packing were preconditioned with $5 \mathrm{~mL}$ of an $8 \mathrm{M}$ aqueous urea solution to deactivate active sites, followed by washes with $5 \mathrm{~mL}$ of methanol and $10 \mathrm{~mL}$ of distilled water. The plasma samples were applied to the columns followed by washes with $4 \mathrm{~mL}$ distilled water and then $4 \mathrm{~mL}$ of $15 \%$ methanol in water. Dexamethasone was eluted with $4 \mathrm{~mL}$ of $70 \%$ methanol in hexane. All solvents were forced through the solid-phase extraction columns at flow rates of about 1-2 drops/s by the use of a vacuum manifold, except for the final elution, which was done by gravity flow to avoid contamination from the vacuum manifold.

\section{Oxidation of Plasma Extracts}

Following removal of the eluting solvent by evaporation under a stream of nitrogen, $1 \mathrm{~mL}$ of $\mathrm{CH}_{2} \mathrm{Cl}_{2}$ was added to each residue with magnetic stirring to promote dissolution of the extracted material. Approximately $10 \mathrm{mg}$ of anhydrous sodium acetate was added to the reconstituted solutions of the extracts; sodium acetate was added to buffer acid liberated during the oxidation. An excess of pyridinium chlorochromate, finely ground with Celite (ca. 1:3 w/w) by using a mortar and pestle, was then added to the solutions. The reaction mixtures were stirred and heated to $60^{\circ} \mathrm{C}$ for $6.5 \mathrm{~h}$.

\section{Removal of Oxidation Reagent}

Solid-phase extraction columns containing $500 \mathrm{mg}$ of silica were prewashed with several column volumes of $\mathrm{CH}_{2} \mathrm{Cl}_{2}$. After completion of the oxidation, the reaction mixtures were transferred directly to the columns, which were then washed with $1.5 \mathrm{~mL}$ of $\mathrm{CH}_{2} \mathrm{Cl}_{2}$. The oxidation product of dexamethasone was eluted with $4 \mathrm{~mL}$ of $5 \%$ acetone in $\mathrm{CH}_{2} \mathrm{Cl}_{2}$. After removal of the $5 \%$ acetone- $\mathrm{CH}_{2} \mathrm{Cl}_{2}$ under nitrogen, the residue was redissolved in $100 \mu \mathrm{L}$ of ethyl acetate. Aliquots of $1-3$ $\mu \mathrm{L}$ were required for analysis by ECNI with GC/MS, GC/MS/MS, or DIP/MS/MS.

\section{Preparation of Calibration Curve}

As an internal standard, $35 \mathrm{ng}$ of ${ }^{13} \mathrm{C}_{6},{ }^{2} \mathrm{H}_{3}$ dexamethasone was added to each of several 1-mL aliquots of Red Cross Blood Bank plasma. Following addition of the internal standard, dexamethasone was added to the $1-\mathrm{mL}$ aliquots in amounts of 0.0 (blank), $0.209,0.418,1.67,4.18,8.36$, and $16.7 \mathrm{ng}$ of dexamethasone in 25-100 $\mu \mathrm{L}$ of methanol. Each standard was prepared in duplicate. The extraction, oxidation, isolation, and analysis procedures for these standards were identical to those used for the clinical plasma samples.

\section{Determination of Accuracy and Precision}

Two aliquots of $1 \mathrm{~mL}$ of blood bank plasma were "spiked" with $35 \mathrm{ng}$ of ${ }^{13} \mathrm{C}_{6},{ }^{2} \mathrm{H}_{3}$-dexamethasone and $2.09 \mathrm{ng}$ of dexamethasone. These duplicate standards were processed independently and analyzed in triplicate by ECNI/MS with GC/MS, GC/MS/MS, and DIP/MS/MS to assess the accuracy and precision of the determination of dexamethasone by these techniques.

\section{Results and Discussion}

Dexamethasone is a synthetic corticosteroid drug that contains one fluorine atom. The structures of dexamethasone and its oxidized 11,17-keto analogue are 


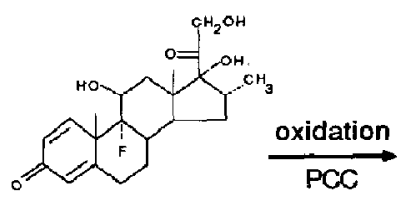

Dexamethasone

Figure 1. Conversion of dexamethasone to its 11,17-keto analogue by chemical oxidation. (9o-nuoro-16a-metmul 1 1: 17 10,21 (9o-huoro-16a-metmy-1 1, 170,21
trial-1, 4 -pregnadien-3,20-dione)

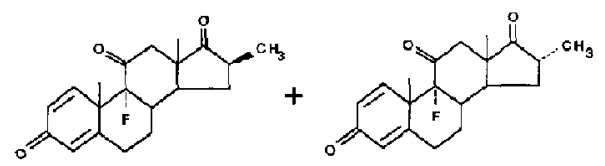

$16 \beta$-methyl-11,17-keto Dexamethasone

$16 \alpha$-methyl-11,17-keto Dexamethasone
(90-thuoro-16B-methyt-1,4androstadiene-3,11,17-trione) (9x-llworo-16a-methyl 1,4androstadiene-3,11,17-trione) depicted in Figure 1. Epimerization of the $16 \alpha-\mathrm{CH}_{3}$ group occurs during the oxidation procedure, producing about $20 \%$ of the $16 \beta-\mathrm{CH}_{3}$ epimer of the oxidation product, $9 \alpha$-fluoro-16-methyl-1,4-androstadiene3,11,17-trione (11,17-keto-dexamethasone). The ECNI spectrum of 11,17 -keto-dexamethasone consists primarily of a peak at $m / z 330$ for the molecular anion and a base peak at $m / z 310$ representing the $[\mathrm{M}-\mathrm{HF}]^{-\cdot}$ ion. A loss of HF is characteristic of compounds containing a fluorine atom.

When the molecular anion of 11,17-keto dexamethasone is chosen as the parent ion and subjected to collision with argon in $Q_{2}$, the predominant fragment is, as expected, $[\mathbf{M}-\mathbf{H F}]^{-}$. The daughter ion spectrum obtained by collision-activated dissociation (CAD) of the molecular anion is shown in Figure 2. Few endogenous compounds contain fluorine; therefore, interfering substances from the plasma matrix are unlikely to undergo a neutral loss of $20 \mathrm{u}$ (loss of HF). Minor peaks are observed at $m / z 295$ and $m / z 280$, which represent $\left[\mathrm{M}-\mathrm{HF}-\mathrm{CH}_{3}\right]^{-}$and $\left[\mathrm{M}-\mathrm{HF}-2 \mathrm{CH}_{3}\right]^{-\cdots}$, respectively. Because the loss of HF is specific for the fluotinecontaining oxidized dexamethasone, CAD conditions were sought that would maximize the detection of ion current at $m / z 310$.

\section{Optimization of MS/MS Parameters}

Two essential MS/MS parameters affecting fragmentation efficiency, $\Sigma F_{i} /\left(\Sigma F_{\mathrm{i}}+P\right)$, and collection efficiency, $\left(\Sigma F_{i}+P\right) / P_{0}$, where $\Sigma F_{i}$ is the sum of the fragment ion intensity, $P$ is the remaining parent ion intensity, and $P_{0}$ is the parent ion intensity without collision gas, are collision gas pressure and collision energy. Several

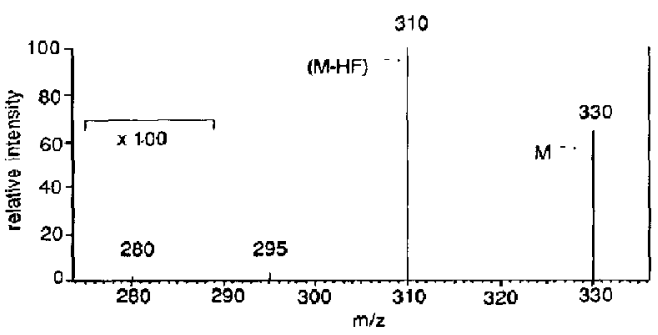

Figure 2. Daughter ion spectrum of the molecular anion of 11,17keto-dexamethasone by CAD [4 eV (Lab), 1.3 mtorr argon]. experiments were designed to dissect out interactive parameters of the $\mathrm{CAD}$ process that could be adjusted individually.

The ion collection efficiency was assessed as a function of collision energy by the experimental protocol described above. The reconstructed TIC, shown in Figure $3 a$, is presented as a function of collision energy at argon pressures of $0.4,0.9$, and 1.3 mtorr in $Q_{2}$. When argon was present at $0.4,0.9$, and 1.3 mtorr in $Q_{2}$, the collection efficiency decreased at collision energies above $3 \mathrm{eV}$; similar attenuation of the TIC at higher collision energy has been noted by other researchers [10]. The poorest collection of ions is observed at the lowest energy examined, $1 \mathrm{eV}$; this is apparently due to the low kinetic energy of the parent ion. The highest collection efficiency occurred with a collision energy of $3 \mathrm{eV}$ for each pressure examined. The value for the

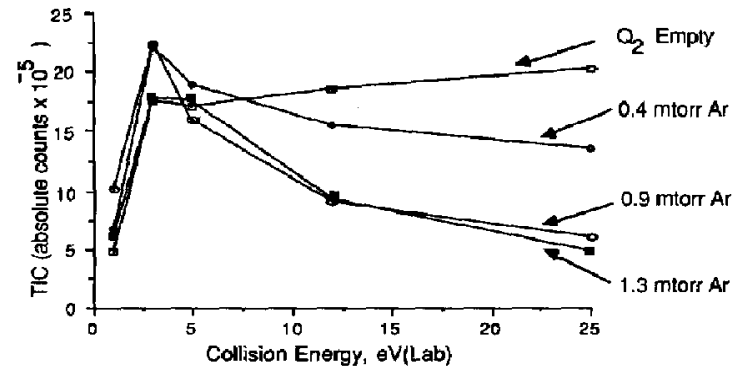

(a)

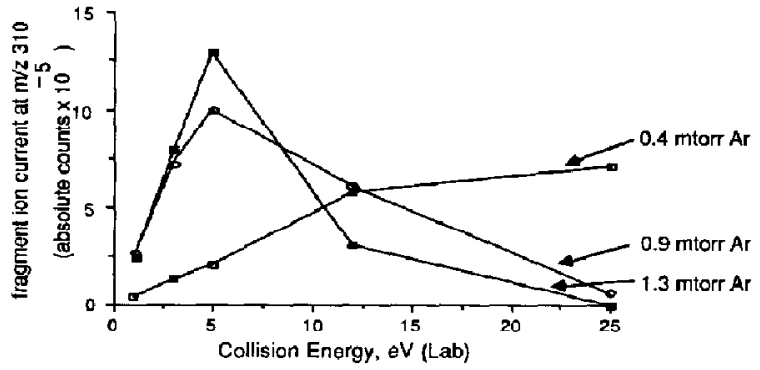

(b)

Figure 3. (a) Reconstructed TIC from CAD of the $M^{-}$of oxidized dexamethasone as a function of collision energy at different collision gas (argon) pressures in $Q_{2}$. (b) Magnitude of $[\mathrm{M}-\mathrm{HF}]^{-}-$daughter ion $(\mathrm{m} / \mathrm{z} 310)$ current as a function of collision energy at three pressures of argon in $Q_{2}$. 
maximum TIC at $3 \mathrm{eV}$ was approximately the same for the three pressures of argon except at 1.3 mtorr, where there was a slight attenuation of the ion beam (about $15 \%)$ due to higher collision gas pressure, and when $Q_{2}$ was empty. We have no reasonable explanation for the TIC results from experiments with no collision gas in $Q_{2}$ being lower than those when $Q_{2}$ is pressurized at collision energies below $5 \mathrm{eV}$; the precision of results ( $\pm 15 \%$ relative standard deviation) is too poor, because of the experimental difficulties in obtaining TIC as a function of both energy and pressure, to distinguish individual values at low energy in Figure $3 a$. Energy effects at 2 mtorr also were examined; considerable reduction in the collection efficiency occurred (about $45 \%$ ) relative to that at other pressures examined (data not shown). In contrast, when the collision cell is empty, a relatively constant ion flux is detected from $3 \mathrm{eV}$ to $25 \mathrm{eV}$, with greatest transmission at 25 $\mathrm{eV}$.

The interdependency of various instrumental parameters involved in an MS/MS experiment makes it difficult to determine what causes the decrease in collection efficiency as collision energy is raised. Of the several variables, greater collision energy increases the fragmentation efficiency, and these daughters may be confined with varying degrees of efficiency in the rfonly field of $Q_{2}[11,12]$. Mass-discriminatory effects are generally pronounced when the daughter ion mass is much lower than the parent mass. In this case, however, the daughter mass $(m / z 310)$ is $94 \%$ of the parent mass ( $m / z 330)$; therefore, losses due to disparities in efficiency of ion containment in $Q_{2}$ should be minimal. Variations in the transmission of ions as a function of collision energy have been noted by Alexander and Boyd [12] for a hybrid BEQQ instrument. In their study, the maximum and minimum transmission of protonated Leu-enkephalin $\left(\mathrm{MH}^{+}, m / z 556\right)$, without collision gas present, varied by a factor of 2 over the collision energy range of $10-30 \mathrm{eV}$ [12]. Fluctuations in the transmission curve for both parent and daughter ions as $E_{L_{a b}}$ increases could account for the apparent losses of ion current. However, in our case with $\mathrm{Q}_{2}$ empty, the constant parent ion flux, which is quite independent of collision energy, would seem to indicate that our losses are not due to an instrumental dependency similar to that of Alexander and Boyd. Thus, by default, target gas pressure facturs appear to cause the decrease in collection efficiency as $E_{\text {Lab }}$ increases. Scattering of both parent and daughter ions may lead to lower transmission into the acceptance region of $Q_{3}$ [13], but the tandem quadrupole configuration is generally considered to be a good focusing device for scattered ions, so scattering losses are probably minor. With increased pressure and higher energies, collision-induced electron detachment [14] may compete with the CAD processes, resulting in formation of undetectable neutral species. All factors described probably contribute to ion losses, but electron detachment is most likely the predominant factor causing the decrease in collection efficiency as collision energy is raised.

The magnitude of fragment ion current at $m / z 310$ as a function of collision energy is represented in Figure 3b. At the lowest pressure, 0.4 mtorr argon, collision at $25 \mathrm{eV}$ provides the greatest amount of $[\mathrm{M}-\mathrm{HF}]^{-}$. At increased pressures, lower energies were required for maximal formation of $[\mathrm{M}-\mathrm{HF}]^{-}$; optimal energy for CAD at 0.9 mtorr and 1.3 mtorr was $5 \mathrm{eV}$. More effective transfer of kinetic energy to internal energy occurs with higher collision frequency [11, 14, 15]; thus, less translational energy is required at higher gas pressures than at low pressure to induce similar fragmentation. Secondary dissociation of $[\mathrm{M}-\mathrm{HF}]^{-}$also may occur at higher pressures; successive losses of $\mathrm{CH}_{3}$. from [M - HF] ${ }^{-}$result in formation of ions of mass 295 and 280 . Peaks at $m / z 295$ and $m / z 280$ increase in intensity as the collision energy is raised at pressures of 0.9 and 1.3 mtorr, whereas the intensity at $m / z 310$ decreases for $[\mathrm{M}-\mathrm{HF}]^{--}$.

As a result of the optimization studies, a pressure of approximately 1.3 mtorr argon in $Q_{2}$ and a collision energy of $4 \mathrm{eV}$ were employed to ensure both high ion collection efficiency and extensive fragmentation of the parent ion of mass 330, resulting in optimal detectability of the daughter ion of mass 310 during the analysis of plasma samples.

\section{SRM vs. SIM}

The improved selectivity of GCISRM over GCISIM is shown in Figure 4, which is a composite of restults from the analysis of a plasma extract for dexamethasone. Shown in Figure $\mathbf{4 a}$ are the selected ion current profiles for the $\mathrm{M}^{-}$and $[\mathrm{M} H \mathrm{HF}]^{--}$of 11,17keto-dexamethasone (top two panels) and 11,17-keto${ }^{13} \mathrm{C}_{6},{ }^{2} \mathrm{H}_{3}$-dexamethasone (bottom two panels); the latter was used as an internal standard during analysis of this plasma sample by SIM. These data were obtained after the extraction and oxidation of a 1.5$\mathrm{mL}$ plasma sample containing $35 \mathrm{ng}$ of internal standard and $0.9 \mathrm{ng}$ of dexamethasone (concentration of $0.6 \mathrm{ng} / \mathrm{mL}$ ). The quantities injected on-column to obtain the data in Figure 4a correspond to approximately $20 \mathrm{pg}$ of oxidized dexamethasone and $0.7 \mathrm{ng}$ of the oxidized isotopically labeled dexamethasone. Shown in Figure $4 \mathrm{~b}$ are the selected reaction current profiles obtained from another aliquot of the same sample as analyzed by GCISRM with CAD of the molecular anions of 11,17-keto-dexamethasone (top panel) and the ${ }^{13} \mathrm{C}_{6},{ }^{2} \mathrm{H}_{3}-11,17-$ keto internal standard (bottom panel). In Figure $4 b$, all the interfering ion current has been eliminated by the MS/MS process. The only signal present is that from the 11,17-keto-dexamethasone and its $16 \beta-\mathrm{CH}_{3}$ epimer (peak at longer retention time). The absence of background makes the determination of the peak height or the integration of the peak area straightforward and increases the precision of replicate analyses. Determination of the baseline position for peak 


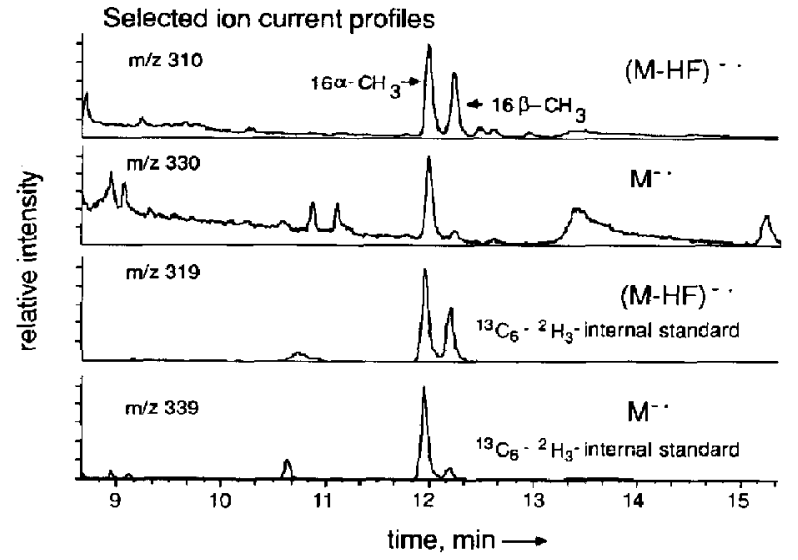

(a)

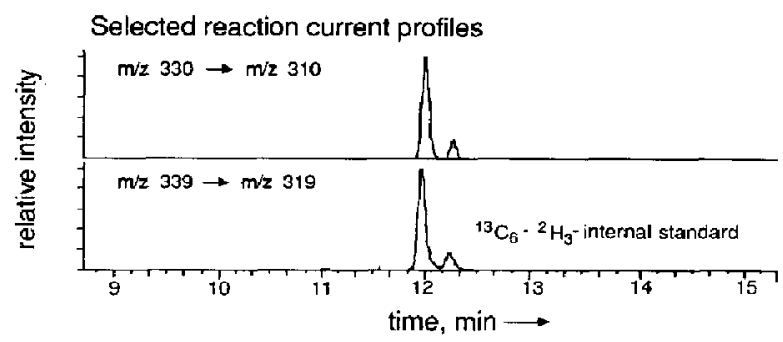

(b)

Figure 4. Comparison of selected ion current profiles obtained by GCIECNI with (a) selected ion monitoring (top two panels for axidized dexamethasone; bottom two panels for internal standard) and (b) selected reaction monitoring (top panel for oxidized dexamethasone; bottom panel for internal standard).

height or area measurement from the SIM data in Figure $4 \mathrm{a}$ is more arbitrary, which leads to poorer precision than that in GCISRM.

Comparison of the calibration curves obtained by SIM and by SRM, shown in Figure 5, indicates that the limits of detection (LOD) of dexamethasone in plasma by the three techniques are identical. The LOD can be defined as the concentration of analyte giving a detector response that is three times the standard deviation of the analytical result for the blank; alternatively, the value of the $y$ intercept of the calibration curve can be used in place of the analytical result for the blank in calculating the LOD [16]. Using this criterion, the LOD of dexamethasone in plasma was $0.25 \mathrm{ng} / \mathrm{mL}$ by SIM ( $\left.y_{\text {int }}=0.026, s=0.0030\right), 0.31 \mathrm{ng} / \mathrm{mL}$ by GC/SKM $\left(y_{\text {int }}=0.029, s=0.0035\right)$, and $0.33 \mathrm{ng} / \mathrm{mL}$ by DIP/SRM $\left(y_{\text {int }}=0.039, s=0.0033\right)$. It is likely that these LODs could be decreased by decreasing the amount of internal standard that is added to the samples. A large amount of ${ }^{13} \mathrm{C}_{6},{ }^{2} \mathrm{H}_{3}$-dexamethasone was added to the plasma samples to serve as a carrier as well as the internal standard. The isotopically labeled internal standard contained about $3 \%$ unlabeled dexamethasone, which resulted in a high blank value. This high blank apparently resulted from contamination of the internal standard prior to its use in these analyses; the blank value in ${ }^{13} \mathrm{C}_{6},{ }^{2} \mathrm{H}_{3}$-dexamethasone should be varishingly low. The high blank was not caused by the oxidation procedure, as a high blank was also observed with conventional EI methodology by the group donating the isotopically labeled material. The detection limit of this assay is limited by the precision with which we can measure the blank as well as the low slope of the calibration curve. The addition of less internal standard should result in a steeper slope for the calibration curve. An experiment was done to determine whether the LOD for dexamethasone in plasma could be decreased by the addition of less internal standard. Standards for a calibration curve were prepared as described in the Experimental section except that one tenth the quantity of internal standard was added to each standard. The limits of detection calculated (as described previously) from the calibration curves obtained during analyses of these standards by GC/SIM and GC/SRM were $0.15 \mathrm{ng} / \mathrm{mL}$ and $0.05 \mathrm{ng} / \mathrm{mL}$, respectively. The lower value for GC/SRM derives from the better precision available from SRM as explained earlier. Peak areas for $m / z 310$ obtained by GC/SRM from standards containing the same amount of dexamethasone indi-

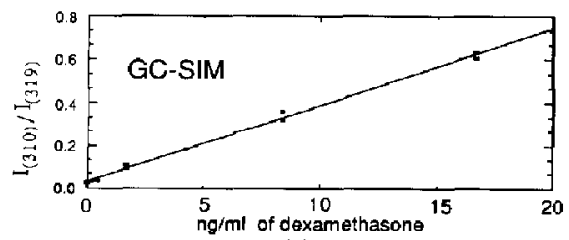

(a)

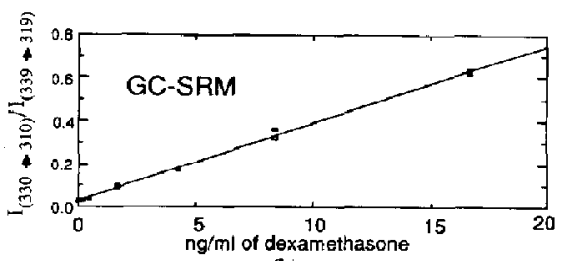

(b)

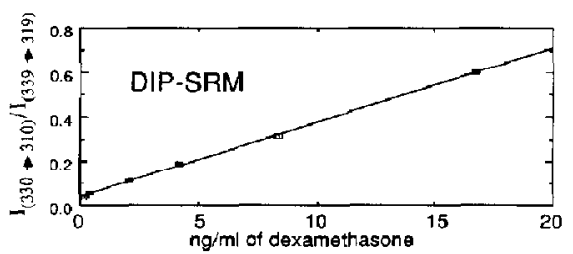

(c)

Figure 5. Calibration curves for the determination of dexamethasone in plasma by (a) GC/ECNI/MS with selected ion monitoring, (b) GC/ECNI/MS/MS with selected reaction monitoring, and (c) DIP/ECNI/MSIMS with selected reaction monitoring. The quantities introduced into the ion source represent a range of 4-200 $\mathrm{pg}$ of dexamethasone and $700 \mathrm{pg}$ of internal standard. 
cated no decrease but small and large amounts of internal standard indicated no decrease in the recovery of dexamethasone when the smaller amount of internal standard was used. Therefore, the smaller amount of internal standard is recommended. In the comparison described above, the LOD for GC/SIM may be high because SIM was carried out in the TSQ70 with $Q_{2}$ and $Q_{3}$ in the rf-only mode, a feature that reduces overall ion transmission by an order of magnitude or more compared to that of a single quadrupole instrument. This was not considered a serious problem, as the central thrust of this report focuses on selectivity and not sensitivity.

Several plasma samples from patients undergoing the dexamethasone suppression test were extracted, oxidized, and analyzed by GC/SIM and by both GC/SRM and DIP/SRM. The quantitative results are shown in Table 1 together with standard deviations that indicate the level of instrumental precision. The results by SIM and SRM with sample introduction by GC are in good agreement with the exception of those for sample P3. The results obtained by SRM with sample introduction by DIP do not differ significantly from those obtained with sample introduction by GC, with the exception of those for samples P3 and P5. GC/SRM is the most selective of the three analytical techniques, because interferences are exciuded by both the GC inlet and MS/MS. Therefore, the results obtained by GC/SRM would be expected to be the most reliable.

Other researchers have reported that a chromatographic step in the sample preparation is necessary in methodology based on ECNI/MS/MS when a general derivatization reagent is used to prepare an electrophilic derivative of the sample [7]. The chromatographic step is required because the electron capture cross sections of many matrix components also are enhanced by the derivatization. The derivatized matrix components can deplete the concentration of thermal electrons in the ion source $[7,17]$. Without suffi-

Table 1. Comparison of GC/ECNI/SIM, GC/ECNI/SRM, and DIP/ECNI/SRM for the determination of dexamethasone in plasma

\begin{tabular}{lccc}
\hline Sample & GC/SIM & GC/SRM & DIP/SRM \\
\hline P2 & $0.63 \pm 0.07(n=2)$ & $0.76 \pm 0.01$ & $0.68 \pm 0.03(n=4)$ \\
P5 & $0.62 \pm 0.09$ & $0.69 \pm 0.02$ & $0.35 \pm 0.01$ \\
P11 & $3.2 \pm 0.4(n=2)$ & $4.0 \pm 0.4$ & $4.4 \pm 0.4$ \\
P18 & $30 \pm 4(n=2)$ & $36 \pm 3$ & $35 \pm 1$ \\
P3 & $0.18 \pm 0.01(n=4)$ & $0.29 \pm 0.01$ & $0.15 \perp 0.01$ \\
7A & $2.1 \pm 0.1$ & $1.9 \pm 0.1$ & $2.11 \pm 0.05$ \\
$7 B^{b}$ & $1.8 \pm 0.1$ & $1.82 \pm 0.04$ & - \\
\hline
\end{tabular}

Concentrations are reported in $\mathrm{ng} / \mathrm{mL}$ of plasma \pm one standard deviation. Number of replicate injections $(m)$ is 3 unless indicated as otherwise.

- The values listed for GC/SIM and DIP/SRM fall below the LOD.

h Duplicate spiked plasma sample $(2.09 \mathrm{ng} / \mathrm{mL})$, measured in triplicate. cient chromatographic separation, the low level of analyte present in the ion source apparently cannot compete effectively with the overwhelming excess of electrophilic components of the matrix for the pool of thermal electrons. However, the chemical oxidation procedure described herein selectively converts the analyte to an electrophilic analogue without greatly enhancing the electrophilic character of the matrix. Therefore, introduction of an oxidized sample by direct probe is viable, because the matrix is less likely to participate in ECNI, and thus, in most cases, it does not hinder detection of the analyte by ECNI.

\section{Conclusions}

The use of selected reaction monitoring for the detection of oxidized dexamethasone in a plasma matrix under ECNI conditions yields results similar to those obtained through the use of SIM. When the GC inlet is used, SRM offers no significant advantage over SIM due to the selectivity already achieved by the chemical oxidation procedure and subsequent ECNI. The additional selectivity of SRM allows the direct probe sample inlet to be used successfully for analyses based on ECNI. Of significant importance is the fact that the nonelectrophilic sample matrix accompanying the oxidized analyte, as prepared by the specialized chemical uxidation procedure described herein, reduces the possibility of quenching the ECNI of the analyte during batch introduction of the sample by the direct probe. An investigation of the effect of structural features on the ECNI response of steroids and cyclic compounds is being pursued.

\section{Acknowledgment}

This work was supported in part by a grant (no. 00480-20) from the Biomedical Research Technology Program of the Division of Research Resources of the National Institutes of Health and from the Michigan State University Agricultural Experiment Station. T. Heath thanks the Yates Memorial Foundation for a fellowship.

\section{References}

1. Her, G. R.; Watson, J. T. Biomed. Environ. Mass Spectrom. 1986, 13, 37-63.

2. Mayer, H., graduate research assistant, Department of Chemistry, Michigan State University, unpublished results.

3. Arana, G. W.; Workman, R. J.; and Baldessarini, R. J. Am. J. Psychiat. 1984, 141, 1619-1621.

4. Kasuya, Y.; Althaus, J. R.; Freeman, J. P.; Mitchum, R. K.; Skelly, J. P. J. Pharm. Sci. 1984, 73, 446-451.

5. Kayganich, K.; Watson, J. T.; Kilts, C; Ritchie, J. Biomed Environ. Mass Spectrom. 1990, in press.

6. Yost, R. A.; Fetterolf, D. D.; Hass, J. R.; Harvan, D. J.; Weston, A. F.; Skotnicki, P. A.; Simon, N. M. Anal. Chem. 1984, 56, 2223-2228. 
7. Johnson, J. V.; Yost, R.; Faull, K. F. Anal. Chem. 1984, 56, $1655-1661$

8. Schweer, H.; Seyberth, H, W.; Meese, C. O.; Fürst, O. Biomed. Environ. Mass Spectrom. 1988, 15, 143-151.

9. Krishriamurthy, T.; Sarver, E. W. Biomed. Environ. Mass Spectrom. 1988, 15, 13-24.

10. Dawson, M.; McGee, C. M.; Brooks, P. M.; Vine, J. H.; Watson, T. R. Biomed. Environ. Mass Spectrom. 1988, 17, 205-211.

11. Dawson, P. H.; French, J. B.; Buckley, J. A.; Douglass, D. J.; Simmons, D. Org. Mass Spectrom. 1982, 17, 205-211.
12. Alexander, A. J.; Boyd, R. K. Int. J. Mass Spectrom. Ion Processes 1989, 90, 211-240.

13. Schey, K. L.; Kenttömaa, H. I.; Wysocki, V. H.; Cooks, R. G. Int. J. Mass Spectrom. Ion Processes 1989, 90, 71-83.

14. Wysocki, V. H.; Kenttömaa, H. I.; Cooks, R. G. Int. J. Mass Spectrom. Iom Processes 1987, 75, 181-208.

15. Dawson, P. H. Int. J. Mass Spectrom. Ion Phys. 1982, 43, 195.

16. ACS Committee on Environmental Improvement, Anal. Chem. 1980, 52, 2242-2249.

17. Hunt, D. F.; Crow, F. W. Anal. Chem. 1978, 50, 1781-1784. 